Questions vives

\section{Questions Vives}

Recherches en éducation

$N^{\circ} 25 \mid 2016$

L'activité des enseignants face au décrochage scolaire : Quelles difficultés et quelles mises en œuvre professionnelles?

\title{
L'enseignant face à la difficulté de la classe : Capacité à agir et décrochage enseignant.
}

Teacher facing the difficulty of class: disempowerment and teacher attrition.

\section{Séraphin Alava}

\section{(2) OpenEdition \\ Journals}

Édition électronique

URL : http://journals.openedition.org/questionsvives/1942

DOI : 10.4000/questionsvives. 1942

ISSN : 1775-433X

\section{Éditeur}

Université Aix-Marseille (AMU)

\section{Édition imprimée}

Date de publication : 7 juillet 2016

ISBN : 978-2-912643-49-0

ISSN : 1635-4079

Référence électronique

Séraphin Alava, «L'enseignant face à la difficulté de la classe : Capacité à agir et décrochage enseignant. », Questions Vives [En ligne], № 25 | 2016, mis en ligne le 27 février 2017, consulté le 10 décembre 2020. URL : http://journals.openedition.org/questionsvives/1942 ; DOI : https://doi.org/ 10.4000/questionsvives. 1942

Ce document a été généré automatiquement le 10 décembre 2020.

\section{(i) $\$$

Questions Vives est mis à disposition selon les termes de la licence Creative Commons Attribution Pas d'Utilisation Commerciale - Pas de Modification 4.0 International. 


\title{
L'enseignant face à la difficulté de la classe : Capacité à agir et décrochage enseignant.
}

Teacher facing the difficulty of class: disempowerment and teacher attrition.

\author{
Séraphin Alava
}

\section{Introduction : l'enseignant face à la difficulté}

1 Au-delà des études assez nombreuses sur l'action d'enseigner et sur les pratiques enseignantes qui décrivent et modélisent les variations des actes didactiques en relation avec l'hétérogénéité des élèves (Altet, 2002 ; Bru, 1992 ; Bru \& Maurice, 2001; Barrère, 2000), on peut repérer dès 1999 des ouvrages qui alertent sur la situation des enseignants et sur les difficultés professionnelles et personnelles qu'ils rencontrent. Parallèlement à la reconnaissance d'une difficulté à enseigner dès 1998, Debarbieux aborde la question de la violence à l'école dans sa dimension enseignante. Le professionnel est alors montré comme victime. L'enseignant est vu dans sa difficulté à gérer ou remédier à des actes violents et des perturbations (Debarbieux, 1996; Janot, 2005 ; Montoya \& Debarbieux, 1998).

2 L'observation des comportements des enseignants face à des difficultés à enseigner est au cœur des travaux de Smylie (1999) qui introduisent le concept de stress professionnel pour parler des difficultés à enseigner. Ce stress et la difficulté croissante des enseignants à adapter leurs stratégies éducatives aux difficultés des élèves et au décrochage peuvent être alors source d'épuisement professionnel ou de burnout (Laugaa, 2004 ; Rudow, 1999). Ces tensions croissantes face aux difficultés conduisent alors les enseignants à adopter des positions, soit de repli professionnel, soit de surinvestissement. La perception d'un mal-être à enseigner va progressivement conduire à la transformation de ces actes professionnels allant parfois vers l'absentéisme (Basco, 2003) et le décrochage par la démission (Romano, 2008). 
Dès 2002, la notion de décrochage enseignant conçue alors que comme l'arrêt de l'activité d'enseignement et la démission est exposée dans les travaux anglo-saxons et canadiens (Borman \& Dowling, 2008 ; Kirsch, 2006 ; Karsenti, Collin, Villeneuve, Dumouchel \& Roy, 2008 ; Stoel \& Thant, 2002). En Europe, ce phénomène est peu étudié en tant que tel mais repris dans des études portant sur le stress et l'épuisement professionnel. Nos recherches visent à élargir la notion de décrochage professionnel en examinant les modalités et le lien entre les phénomènes de retrait progressif de l'enseignant de l'activité professionnelle afin de comprendre comment l'enseignant, face aux difficultés de la classe, perçoit un empêchement professionnel dans l'accomplissement de ses compétences et comment l'enseignant réagit à cet empêchement.

4 À partir d'une étude de terrain menée auprès des enseignants des écoles primaires et secondaires de Midi-Pyrénées (640 enseignants), nous avons voulu aborder les relations existantes entre les difficultés vécues par les enseignants dans la gestion des élèves décrocheurs et les situations réelles de fatigue, de démotivation, de retrait professionnel des enseignants. Notre approche quantitativiste a pour but de faire émerger les invariants de ce repli et de comprendre les corrélations qui existent entre ces phénomènes d'absentéisme, de retrait professionnel et la perception subjective par l'enseignant des composantes de son activité professionnelle. Afin d'identifier précisément le stress, l'enquête par questionnaire conduite en relation avec le Rectorat de Toulouse utilise l'épuisement des échelles standardisées de Karasek (Karasek et al., 1998) et de Maslach (Maslach, Jackson \& Leiter, 1996). Nous avons pareillement construit une échelle du décrochage enseignant que nous cherchons à valider et à améliorer. Notre approche vise à comprendre et expliquer les processus d'engagement et de désengagement professionnel dans la gestion des élèves en difficulté. Parfois, l'enseignant sentant sa capacité d'agir remise en cause, percevant des difficultés réelles à se faire entendre, se met en retrait et décroche. Nos résultats permettent de mieux percevoir les processus psychologiques mis en jeu dans l'apparition de ce phénomène.

5 Nous définissons le décrochage professionnel de l'enseignant comme l'arrêt de l'activité en référence aux travaux de Karsenti (Karsenti, Collin, Villeneuve, Dumouchel \& Roy, 2008); Ndoreraho \& Martineau, 2006). Puis à la suite des travaux de Pécout et Méard (2014), nous examinons le comportement des enseignants face à l'accrochage-décrochage des élèves pour montrer que, dans certaines situations, l'enseignant se met en retrait de son activité ou perçoit un sentiment personnel d'empêchement à travailler. Ce phénomène que nous pourrions nommer "décrochage silencieux" s'appuie sur les travaux de Laplante et de Tougas (2011) portant sur le désengagement professionnel des infirmiers et d'autres professionnels en situations de stress. Le décrochage professionnel est donc pour nous un continuum de pratiques adaptatives de l'enseignant à la gestion de situations professionnelles à risque, allant de l'incapacitation (perte du pouvoir d'agir) au désengagement et au décrochage par abandon.

\section{Enseignant en situation professionnelle difficile}

Placé au cœur du dispositif enseignement-apprentissage, clé de voûte de l'action didactique, l'enseignant est paradoxalement peu «approché » dans sa dimension humaine et professionnelle. Enseignant en action, il conduit des activités pédagogiques, vit au sein de la classe et de l'établissement, agit, interagit au quotidien et vit au travail 
dans des contextes techniques, humains et sociaux qui ont un effet sur les conditions de son travail.

Dès les premiers travaux de recherche sur la professionnalisation des enseignants et sur l'acte d'enseigner (Bru, 1992) la question des situations difficiles est au cœur de l'étude de la «variabilité didactique ». La question est alors de comprendre comment l'enseignant fait varier ses choix et ses actes professionnels face à l'hétérogénéité des élèves et aux situations d'échec. Les effets des conditions de vie au travail sur l'action de l'enseignant et l'étude des interactions élèves-maitre sont d'abord abordés sous l'angle pédagogique ou didactique afin de préciser, décrire et modéliser l'action de l'enseignant. Dans cette même perspective d'études des pratiques enseignantes et de leurs liens avec la difficulté ou la réussite de l'élève, les travaux de Bressoux puis de Duru Bellat (Bressoux, 1994 ; Duru Bellat \& Mingat, 1997) montrent des relations dans les deux sens entre les conditions de travail enseignant et les réussites scolaires.

Dans cet effort de compréhension de l'acte d'enseigner, les actions de l'enseignant sont référencées au regard des compétences nécessaires à l'accomplissement de son action. L'enseignant dans sa dimension personnelle, psychologique, médicale est alors peu ou pas pris en compte. Pourtant l'enseignant, comme tout humain au travail, agit et interagit avec les milieux, les acteurs, l'environnement proche. Sur ce point, les travaux américains (Woods, 1977; Smylie, 1999), les recherches d'Huberman (1993) montrent que l'enseignant, tout en étant un professionnel compétent, subit stress et pression propres à modifier son comportement. Ces interactions produisent des effets sur sa santé, son action et parfois sur son état général. L'enseignant comme tout professionnel vit au travail, il y conduit des actions et structure une expérience de vie. Ces conditions de travail, celles que les chercheurs appellent «le contexte d'action", vont agir sur la dimension pédagogique et éducative de l'activité mais aussi sur la personne agissante. D'autres travaux de recherche (Galloway, Pankhurst, Boswell \& Green, 1984 ; Laughlin, 1984) montrent ainsi un professionnel impuissant, «subissant» les conditions de travail et les difficultés de la classe. Enseigner est alors analysé comme une activité stressante (Van Horn, Schaufeli, Greenglass \& Burke, 1997; Wisniewski \& Gargiulo, 1997; Borg, 1990), une activité mettant en jeu les affects de l'enseignant et pouvant perturber la santé du professionnel (Kyriacou, 1987 ; Dunham, 1992 ; Farber, 1984).

Par ailleurs, dans les études sur le climat et la violence scolaires (Blin, 2001 ; Debarbieux, 1996 ; Montoya \& Debarbieux, 1998), les résultats montrent que les enseignants gèrent du mal-être, des «chocs " perturbant profondément leurs actions professionnelles. Ces perturbations au quotidien sont de nature à modifier profondément les actions professionnelles. En réponse aux perturbations, l'enseignant réagit en modifiant le système complexe d'interrelations entre ses pensées, ses émotions et ses comportements pouvant aller jusqu'à l'impossibilité d'agir face à la situation (Morcilla, Martin \& Blin, 2004).

\section{De la difficulté professionnelle au décrochage enseignant}

Face aux difficultés d'enseigner, au manque de reconnaissance sociale ou professionnelle, face aux violences subies verbales ou physiques, l'enseignant va développer des stratégies de résistance, d'adaptation ou de sauvegarde. Dès les années 2000, de nombreux 
chercheurs notent l'apparition de souffrance réelle et d'épuisement chez les enseignants (Rudow, 1999; Laugaa, 2004). Cette situation de mal-être professionnel est source d'un malaise chez les enseignants (Gendron, 2006). Maleyrot (2012) parle alors dans certains cas de rupture identitaire des enseignants. Que l'on analyse cette situation comme étant la résultante d'un malaise, d'une incompréhension ou de prolétarisation des enseignants (Vergne, 2010), il existe au début du $\mathrm{xxI}^{\mathrm{e}}$ siècle une situation importante de décalage entre les horizons d'attente des enseignants et la réalité professionnelle. Ces écarts produisent des effets variables qui vont de la redéfinition que l'on se fait du métier, en passant par le sentiment d'être empêché à agir professionnellement jusqu'au décrochage professionnel par la démission.

11 Karsenti, Collin, Villeneuve, Dumouchel et Roy (2008) parlent alors de décrochage enseignant pour marquer les situations croissantes d'abandon du métier par démission. Cette notion de décrochage enseignant ou décrochage professionnel qui apparaît autour des années 2000 aux USA et au Canada marque d'abord dans un premier temps la simple analyse des démissions ou de ruptures de contrats par des enseignants. Dès 1991, Macdonald étudie les départs prématurés de la carrière et analyse les causes à la fois de ce départ et du malaise des enseignants. Ingersoll (2001) et Stoel et Thant (2002) apportent des informations scientifiques sur les raisons de ce mal-être enseignant et l'impact de ces décrochages. Les causes sont diverses, certaines les définissant à partir du processus de burnout: charge de travail (Chaplain, 2008; Kirsh 2006), gestion de classe difficile (Romano, 2008), mauvaises conditions de travail (Guarino, Santibanez \& Daley, 2006), mauvaises relations au sein de l'établissement (Borman \& Downling, 2008).

En référence à d'autres approches, le décrochage peut prendre un autre sens, pouvant être compris à partir de la notion d'empêchement à agir (Lecuivre \& Roger, 2012) ou d'empêchement à penser des enseignants (Ourghanlian, 2011). Mes travaux prolongent cet élargissement du concept de décrochage pour parler d'un ensemble d'actions et de processus qui accompagne le retrait de l'enseignant.

Face à la difficulté à agir, l'enseignant perd le sentiment d'autonomie dans ses actes et dans sa capacité à agir. Les chercheurs ont défini le concept de "disempowerment" comme le sentiment construit par l'enseignant de son incompétence à atteindre les buts pédagogiques qu'il se fixe ou que l'institution lui fixe (Bolin, 1989; Sprague, 1992; Talbert, 2003). Le sentiment de son incapacité à agir efficacement (Talbert, 2003) produit alors un sentiment de marginalisation de l'enseignant par rapport à ses horizons d'attente et parfois par rapport à ses pairs. Le sentiment de "disempowerment" est aussi en lien avec une difficulté à construire des initiatives et à se percevoir comme efficace dans ses nouvelles actions. L'enseignant alors perd son autonomie au sens qu'il retarde ses initiatives et se met en retrait des situations (Davies, Fulop, Hutchings, Ross, \& Berkics, 2004 ; Yamashita, 2006)

\section{Méthodologie}

Notre recherche s'appuie sur une enquête de terrain menée en 2013 auprès des enseignants des écoles primaires et secondaires de Midi-Pyrénées (497 enseignants).

- Population : 497 enseignants dont 122 hommes et 375 femmes. Ces enseignants enseignent au premier degré, au collège et au lycée. Ce sont des enseignants titulaires et non-titulaires. Pour améliorer la représentativité de notre échantillon, nous avons 
procédé à un redressement de la population sur les trois variables sexe, niveau d'établissement, statut titulaire ou non.

- Méthodologie : Questionnaire en ligne Limesurvey - période avril mai juin 2013. Le questionnaire comportait les domaines suivants :

- Variables d'identification

- Échelle de mesure du climat d'établissement adapté à partir des échelles de Fotinos (2012) et Debarbieux (2009)

- Échelle de Maslach sur le burnout « Maslach Burnout Inventory, 1986»

- Échelle de Karasek sur la Qualité de vie au travail « Job Content Questionnaire, 1988 »

- Échelle subjective de fatigue physique, morale et nerveuse

- Échelle des indicateurs de décrochage scolaire (construite pour l'enquête).

Nous avons voulu aborder les relations existantes entre les difficultés vécues par les enseignants dans la gestion des élèves décrocheurs et les situations réelles de démotivation, retrait professionnel des enseignants. Dans notre enquête, nous avons identifié les élèves décrocheurs par quatre critères (taux d'absentéisme, décrochage silencieux, niveau de perturbation en classe, difficultés de l'élève à réaliser les activités attendues durant une séance). Notre approche quantitativiste a pour but de de comprendre les corrélations qui existent entre ces phénomènes d'absentéisme et de retrait professionnel. Nous avons travaillé avec les logiciels XLSTAT et SPSS. Notre démarche statistique a cherché à identifier les corrélations, les typologies d'usages et à mesurer le rapport de chances ou de risques - odds ratio - entre les variables corrélées. L'analyse des données (tri à plat, test de corrélation de Pearson, tableau de contingence avec calcul des odds ratios) a permis de préciser les niveaux de corrélations (coefficient de Pearson et valeur-p) entre les variables et le rapport de chances (odds ratio) de l'évolution d'une variable par rapport à une autre.

En effet, l'odds ratio (OR) est une statistique qui permet d'identifier le rapport de chances ou de risques (si l'évènement dont l'apparition statistique est mesuré est considéré comme négatif) qu'un événement apparaisse si nous passons d'une population $\mathrm{A}$ à une population B. Cet OR est le rapport des cotes (la cote d'un événement $E$ est $p(E) /(1-p(E)$ ). Par exemple, l'OR de l'événement $\mathrm{E}$ «avoir un niveau de décrochage supérieur à la moyenne » chez les enseignants titulaires par rapport aux enseignants remplaçants par exemple est le rapport de la cote de $\mathrm{E}$ chez les enseignants titulaires par rapport à la cote de $\mathrm{E}$ chez les enseignants remplaçants.

Pour mesurer les effets de la difficulté à gérer certaines situations en classe, nous avons construit une échelle du décrochage enseignant. Pour construire cette échelle, nous avons en premier lieu examiné 20 études portant sur la thématique les risques psychosociaux. Cette recension montre que les outils pour l'évaluation des risques physiques, psychologiques existent et sont aujourd'hui assez bien standardisés.

Tableau 1 : Inventaire des recherches portant sur la qualité de vie au travail des enseignants

\begin{tabular}{|l|l|l|}
\hline Enquête & Outils de mesure & Année \\
\hline Maslach et Jackson & Maslach Burnout Inventory & 1986 \\
\hline Finlay-Jones & General Health Questionnaire (GHQ) & 1996 \\
\hline V. Kovess et al & Questionnaire MGEN & 1999 \\
\hline
\end{tabular}




\begin{tabular}{|l|l|l|}
\hline Denis Jeffrey & $\begin{array}{l}\text { Échelle personnelle construite sur la base du test de Maslash, } \\
\text { Susan et Michael }\end{array}$ & 2002 \\
\hline G. Jaoul, V. Kovess, & Échelle Ilfeld dérivée de la HSC & 2002 \\
\hline Janosz & Questionnaire sur l'Environnement Socio-éducatif (QES, Janosz) & 2002 \\
\hline J. Sangsue, G.Vorpe & Questionnaire de Janosz adapté (Janosz, et al., 1998) & 2004 \\
\hline J. Sangsue, G.Vorpe & Questionnaire développé par Travers et Cooper (1996) & 2004 \\
\hline J. Sangsue, G.Vorpe & $\begin{array}{l}\text { Version française du CES-D de Radloff, 1977(Center for } \\
\text { Epidemiologic Studies Depression Scale) }\end{array}$ & 2004 \\
\hline $\begin{array}{l}\text { Laugaa et Bruchon- } \\
\text { Schweitzer }\end{array}$ & Échelle de coping de Dewe (1985). & 2005 \\
\hline $\begin{array}{l}\text { Laugaa et Bruchon- } \\
\text { Schweitzer }\end{array}$ & Échelle à 10 items de Jerusalem et Schwarzer (1992) & 2005 \\
\hline $\begin{array}{l}\text { Laugaa et Bruchon- } \\
\text { Schweitzer }\end{array}$ & Échelle M.B.I. de Maslach et Jackson (1984) & 20010 \\
\hline Fotinos MGEN & Questionnaire d'enquête « Climat scolaire » & 2005 \\
\hline P.A. Genoud, \& al. & Version française du Maslach Burnout Inventory & 2006 \\
\hline CSEE & Questionnaire de mesure du stress & 2006 \\
\hline Karsenti et Collin & Questionnaire sur les raisons du décrochage & 2011 \\
\hline Houlfort et Sauvé & Questionnaire symptômes de détresse & 2009 \\
\hline Houlfort et Sauvé & Questionnaire mesure du stress et de la fatigue & 2010 \\
\hline Société des agrégés & Questionnaire sur les raisons du décrochage professionnel & 2 \\
\hline Fotinos \& Horenstein & Questionnaire d'enquête « QVT » & 2005 \\
\hline
\end{tabular}

De ces questionnaires et échelles, nous avons retenu 22 items d'effets que nous avons testés auprès de 40 enseignants afin qu'ils nous précisent la fréquence et la force d'apparition de ces items.

- Difficultés de travail

- Fatigue au travail

- Niveau de stress au travail

- Fatigue morale excessive

- Mésestime de soi

- Sentiment d'empêchement à agir

- Baisse du sentiment d'efficacité

- Sentiment de retrait professionnel

- Routinisation volontaire

- Dégradation de sa vision personnelle du métier

- Retard - oubli

- Phobie de classe, de travail

- Fermeture relationnelle

- Congés maladie

- Epuisement - troubles physiques

- Envie de démission - de changement 
- Congés de courte ou longue durée

- Démarche de reconversion

- Troubles graves associés

- Burnout

- Dépression

- Suicide

C'est cette échelle qui a servi de bases à la mesure du décrochage enseignant. Les entretiens ainsi que le test de l'échelle ont permis de repérer plusieurs sous-échelles.

Schéma 1 : Composantes de l'échelle de décrochage professionnel.

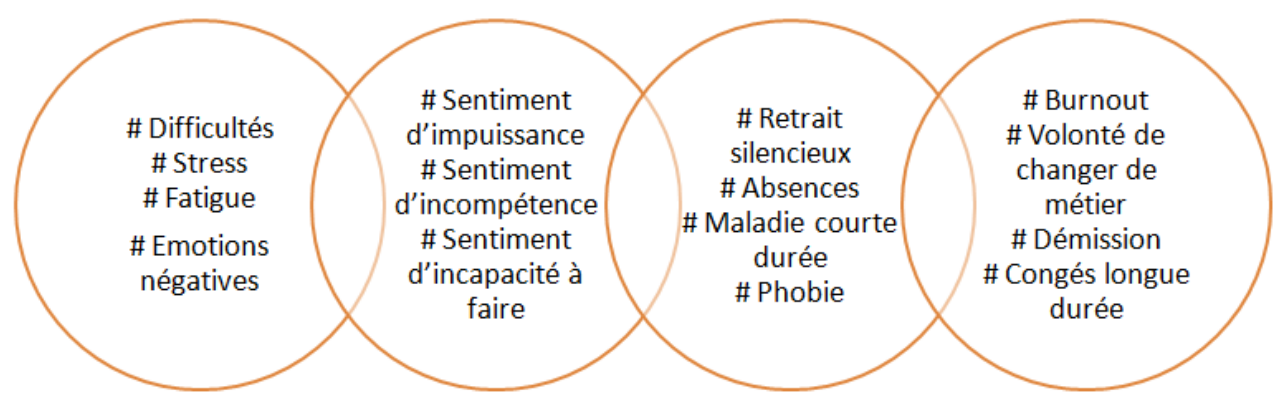

Enfin, en respectant les grilles de notation du test de Malash et de Karasek, nous avons mis ces résultats en relation avec l'indice de décrochage construit à partir des items de l'échelle du décrochage enseignant.

En synthèse, le test de Malasch a permis de recueillir la valence de chaque personne face au burnout ; celui proposé par Karasek a été utilisé pour repérer la perception que chaque individu pouvait avoir de son stress. Enfin, l'échelle du décrochage professionnel a été mobilisée pour recueillir des informations sur le ressenti des enseignants confrontés aux difficultés professionnelles. Ce sont les corrélations entre ces trois variables qui contribuent à mettre en avant certains résultats sur le décrochage professionnel des enseignants.

\section{Résultats}

Dans l'objectif de mesurer les liens existant entre stress, fatigue au travail et décrochage scolaire, nous avons effectué trois mesures complémentaires auprès des enseignants de notre enquête.

\subsection{Décrochage professionnel des enseignants}

Interrogés sur les effets des difficultés scolaires sur leurs pratiques professionnelles, les enseignants en majorité mettent en avant la fatigue et le sentiment de ne pouvoir réaliser leur métier. Sur l'ensemble des résultats de cette échelle, nous identifions des sousgroupes qui expriment des effets en matière de santé, des effets psychologiques et des effets liés au sentiment d'incapacité à agir. 
Tableau 2 : Étude des effets chez l'enseignant des difficultés scolaires (Nous avons regroupé les items des trois composantes par des codes lettrés de A à D)

\begin{tabular}{|l|l|l|}
\hline Échantillon & Codes & $\%$ \\
\hline Les difficultés provoquent chez moi des retards et des oublis & A & 14,5 \\
\hline Les difficultés me rendent malade & B & 16,1 \\
\hline Les difficultés me donnent envie de laisser filer & A & 24,2 \\
\hline Les difficultés perturbent ma vie personnelle & C & 27,4 \\
\hline Les difficultés agissent sur mon estime professionnelle & C & 30,6 \\
\hline Les difficultés me donnent envie de changer de métier & A & 30,6 \\
\hline Les difficultés me désarment & A & 32,3 \\
\hline Les difficultés m'empêchent de prendre des risques & A & 37,1 \\
\hline Les difficultés me perturbent pour la journée & C & 38,7 \\
\hline Les difficultés détériorent la vision de mon métier & A & 39,5 \\
\hline Les difficultés ralentissent ma capacité d'innovation & A & 41,9 \\
\hline Les difficultés ont sur moi un effet démotivant pour vous & C & 42,7 \\
\hline Les difficultés ont une influence sur ma santé & B & 45,2 \\
\hline Les difficultés m'empêchent d'accomplir le travail que je souhaitais faire & D & 51,6 \\
\hline Les difficultés rendent plus difficile la réussite de mon métier & D & 54,0 \\
\hline Les difficultés me fatiguent & B & 65,3 \\
\hline
\end{tabular}

Nous avons ensuite regroupé les items de l'échelle de décrochage professionnel suivant trois axes (santé, psychologique, incapacitation). Après nous avons regroupé l'échelle de Likert en trois zones (peu, moyen, beaucoup). Nous constatons que les effets les plus importants portent sur la santé et sur le sentiment d'empêchement au travail.

Tableau 3 : Typologie des effets chez l'enseignant des difficultés scolaires

\begin{tabular}{|l|l|l|l|l|}
\hline & Codes & Peu & Moyen & Beaucoup \\
\hline Effets sur la santé & B & 47,6 & 50,0 & 41,1 \\
\hline Effets psychologiques & C & 36,3 & 27,4 & 24,2 \\
\hline Effets d'incapacitation & A & 16,1 & 22,6 & 34,7 \\
\hline
\end{tabular}

Dans ce tableau, lorsque nous examinons la colonne où l'effet de décrochage est peu perçu par le sujet, nous constatons un haut niveau d'effet sur la santé, puis quand nous examinons la colonne «beaucoup », alors l'effet d'incapacitation arrive en second niveau devant les effets psychologiques. La santé (fatigue, troubles chroniques) sont les premiers impactés par le décrochage.

Pour mieux identifier le niveau de décrochage professionnel, nous transformons les résultats donnés (échelle de Likert) par les sujets en note pour calculer pour chaque 
individu un indice de décrochage. Nous regroupons ensuite les items en deux groupes d'items : décrochage silencieux par exemple «les difficultés me donnent envie de laisser filer » ou décrochage visible « les difficultés me rendent malade ».

Tableau 4 : Indice du décrochage enseignant (note sur 10)

\begin{tabular}{|l|l|l|l|}
\hline & Indice de décrochage & Décrochage silencieux & Décrochage visible \\
\hline moins de 2 & 38,0 & 36,3 & 43,6 \\
\hline 2 à 5 & 27,3 & 12,1 & 28,2 \\
\hline de 5 à 7 & 18,6 & 14,5 & 17,7 \\
\hline plus de 7 & 16,2 & 37,1 & 10,5 \\
\hline Au dessus de la moyenne & 34,7 & $\mathbf{5 1 , 6}$ & 28,2 \\
\hline
\end{tabular}

Les résultats montrent que le décrochage le plus important est d'abord silencieux. Le test de corrélation (matrice de Pearson) entre les variables d'identification et les décrochages (nous regardons ici l'indice de décrochage en dessous de la moyenne) montre des liens significatifs.

Tableau 5 : Test de corrélation (matrice de Pearson) entre les variables d'identification et les niveaux de décrochage.

\begin{tabular}{|l|l|l|l|}
\hline Variables & $\begin{array}{l}\text { Décrochage au } \\
\text { dessus de la } \\
\text { moyenne }\end{array}$ & $\begin{array}{l}\text { Décrochage silencieux } \\
\text { dessus de la } \\
\text { moyenne }\end{array}$ & $\begin{array}{l}\text { Décrochage visible au } \\
\text { dessus de la moyenne }\end{array}$ \\
\hline Type d'établissement : & $\mathbf{0 , 1 1 4}$ & 0,058 & $\mathbf{0 , 1 4 6}$ \\
\hline Sexe : & $-0,014$ & 0,036 & $\mathbf{- 0 , 0 8 7}$ \\
\hline Age : & 0,047 & $-0,018$ & $\mathbf{0 , 1 1 3}$ \\
\hline Etudes : & 0,036 & $-0,019$ & $-0,010$ \\
\hline Ancienneté : & $-0,006$ & $-0,002$ & 0,034 \\
\hline $\begin{array}{l}\text { Vous êtes sur un poste } \\
\text { d'adjoint à titre définitif }\end{array}$ & $\mathbf{- 0 , 1 2 3}$ & $\mathbf{- 0 , 1 1 3}$ & $\mathbf{- 0 , 1 5 7}$ \\
\hline $\begin{array}{l}\text { Vous êtes sur un poste de } \\
\text { remplacements }\end{array}$ & 0,044 & $\mathbf{0 , 0 8 3}$ & $-0,011$ \\
\hline $\begin{array}{l}\text { Vous êtes sur un poste } \\
\text { d'enseignants spécialisés }\end{array}$ & $\mathbf{0 , 1 1 3}$ & $\mathbf{0 , 1 5 7}$ & $\mathbf{0 , 0 9 2}$ \\
\hline
\end{tabular}

Les valeurs en gras sont différentes de 0 à un niveau de signification alpha =0,5

Les niveaux de corrélation au seuil significatif de 0,05 montrent que :

- Les collèges sont aujourd'hui les lieux où le décrochage professionnel est le plus important.

- Les femmes ont des niveaux de décrochage visible moins importants que ceux des hommes.

- Plus on est âgé et plus on a un indice de décrochage visible au-dessus de la moyenne. 
31 La situation professionnelle est en corrélation avec le décrochage : être adjoint protège du décrochage, être remplaçant est corrélé avec un risque plus fort de décrochage silencieux, être enseignant spécialisé est corrélé avec un risque général de décrochage.

Pour poursuivre nos analyses, nous avons cherché à repérer les sources de fragilisation des conditions de travail en vérifiant leurs niveaux de corrélation avec le décrochage et ses modalités silencieux ou visibles

Tableau 6 : Test de corrélation (matrice de Pearson) entre les sources de fragilisation de la qualité de vie au travail et les niveaux de décrochage

\begin{tabular}{|c|c|c|c|}
\hline Variables & $\begin{array}{l}\text { Décrochage } \\
\begin{array}{ll}\text { dessus de la } \\
\text { moyenne }\end{array}\end{array}$ & 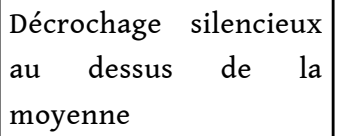 & $\begin{array}{l}\text { Décrochage visible au } \\
\text { dessus de la moyenne }\end{array}$ \\
\hline $\begin{array}{l}\text { Changement des } \\
\text { conditions de travail }\end{array}$ & 0,298 & 0,354 & 0,262 \\
\hline $\begin{array}{l}\text { L'allongement de } \\
\text { l'activité }\end{array}$ & $-0,007$ & 0,014 & 0,011 \\
\hline $\begin{array}{l}\text { La non reconnaissance } \\
\text { de votre travail }\end{array}$ & 0,346 & 0,356 & 0,289 \\
\hline $\begin{array}{l}\text { L'augmentation des } \\
\text { responsabilités }\end{array}$ & 0,014 & 0,044 & $-0,003$ \\
\hline $\begin{array}{l}\text { La dégradation du } \\
\text { climat en classe }\end{array}$ & 0,604 & 0,538 & 0,458 \\
\hline $\begin{array}{l}\text { Le changement des } \\
\text { programmes }\end{array}$ & 0,174 & $-0,012$ & 0,240 \\
\hline $\begin{array}{l}\text { L'augmentation } \quad \mathrm{du} \\
\text { nombre d'élèves }\end{array}$ & 0,095 & 0,128 & 0,141 \\
\hline $\begin{array}{l}\text { La dégradation de la } \\
\text { relation aux parents }\end{array}$ & 0,221 & 0,132 & 0,148 \\
\hline
\end{tabular}

33 Les résultats du test de corrélation (matrice de Pearson) montrent que c'est la dégradation du climat de classe $(0,604-0,538-0,458)$ qui est l'élément le plus corrélé. Nous trouvons ensuite la non-reconnaissance du travail de l'enseignant (0,346 - 0,356 $0,280)$, le changement des conditions de travail $(0,298-0,354-0,262)$. Viennent ensuite la dégradation des relations avec les parents et les changements de programmes.

34 Nous avons poursuivi les analyses en recherchant des sources de fragilisation dans le sentiment de maîtrise par les enseignants des quatre items (maîtrise disciplinaire, maîtrise pédagogique, maîtrise des bruits et incivilités, maîtrise des violences).

Tableau 7 : Tri à plat des niveaux de maîtrise des enseignants

\begin{tabular}{|l|l|l|l|l|}
\hline & $\begin{array}{l}\text { Maîtrise } \\
\text { disciplinaire }\end{array}$ & $\begin{array}{l}\text { Maîtrise } \\
\text { pédagogique }\end{array}$ & $\begin{array}{l}\text { Maîtrise du } \\
\text { bruit }\end{array}$ & $\begin{array}{l}\text { Maîtrise de la } \\
\text { violence }\end{array}$ \\
\hline $\begin{array}{l}\text { En dessous de la } \\
\text { moyenne }\end{array}$ & 30,6 & 33,1 & 45,2 & 50 \\
\hline
\end{tabular}




\begin{tabular}{|ll|l|l|l|l|}
\hline $\begin{array}{l}\text { Au-dessus de la } \\
\text { moyenne }\end{array}$ & 69,4 & 66,9 & 54,8 & 50 \\
\hline
\end{tabular}

Le calcul des corrélations montre de façon significative qu'un faible niveau de maîtrise de ces 4 items est corrélé (au seuil alpha de 0,05) avec un niveau de décrochage au-dessus de la moyenne. La mesure grâce au test de Fisher de l'odds ratio montre que les maîtrises disciplinaires $(O R-35,32)$ et pédagogiques $(O R-11,78)$ sont des éléments permettant d'éviter le décrochage professionnel.

Tableau 8 : Test de corrélation (matrice de Pearson) et calcul des odds ratio entre le faible niveau de maitrise et le niveau de décrochage moyen

\begin{tabular}{|l|l|l|}
\hline & $\begin{array}{l}\text { Décrochage au-dessus de la } \\
\text { moyenne }\end{array}$ & $\begin{array}{l}\text { Décrochage au-dessus de la } \\
\text { moyenne }\end{array}$ \\
\hline $\begin{array}{l}\text { Maible niveau maitrise de Pearson } \\
\text { disciplinaire }\end{array}$ & $\mathbf{0 , 4 5}$ & 35,32 \\
\hline $\begin{array}{l}\text { Faible niveau maitrise } \\
\text { pédagogique }\end{array}$ & $\mathbf{0 , 4 0}$ & 11,78 \\
\hline $\begin{array}{l}\text { Faible niveau maitrise bruits } \\
\text { incivilités }\end{array}$ & $\mathbf{0 , 2 2}$ & 2,61 \\
\hline Faible niveau maitrise violences & $\mathbf{0 , 1 9}$ & 2,22 \\
\hline
\end{tabular}

Les valeurs en gras sont différentes de 0 à un niveau de signification alpha =0,5

\subsection{Maslach Burnout Inventory}

Le MBI explore trois versants de la fatigue au travail : l'épuisement, la dépersonnalisation, l'accomplissement personnel. C'est l'échelle internationale standard pour l'étude du burnout (état dépressif lié au milieu professionnel). Cet état dépressif se déclenche suite à une exposition constante et prolongée au stress du travail. Il est plus généralement associé aux métiers à forte sollicitation mentale, émotionnelle et affective, aux postes à responsabilités ou encore lorsque les objectifs sont difficiles voire irréalisables.

- Épuisement professionnel (score inférieur à $17:$ burnout bas, entre 18 et 29 : burnout moyen, au-dessus de 30 : burnout élevé)

- Dépersonnalisation (score inférieur a 5 : burnout bas, entre 6 et 11 : burnout moyen, audessus de 12 : burnout élevé)

- Accomplissement personnel (score supérieur à 40 : burnout bas, entre 34 et 39 : burnout moyen, inférieur à 33 : burnout élevé

Tableau 9 : résultat du test Maslash MBI

\begin{tabular}{|l|l|l|l|}
\hline & Faible (\%) & Modéré (\%) & Fort (\%) \\
\hline Score d'accomplissement personnel & 43,4 & 32,9 & 23,7 \\
\hline Épuisement professionnel & 14,5 & 44,7 & 40,8 \\
\hline
\end{tabular}




\begin{tabular}{|l|l|l|l|}
\hline Dépersonnalisation & 35,5 & 48,7 & 15,8 \\
\hline
\end{tabular}

37 L'échelle Maslash indique un risque important de burnout (épuisement fort, dépersonnalisation forte, accomplissement faible). Dans notre enquête, ce risque est de $6,6 \%$ pour une prévalence forte et de $26,3 \%$ pour une prévalence moyenne et forte ; ce niveau est légèrement plus bas que celui constaté dans les études européennes qui identifient une prévalence à un burnout élevé de 9,3\% chez les enseignants et de 9,9\% chez les non enseignants (Jaoul, Kovess, 2004).

Tableau 10 : Niveau du risque de burnout dans la population

\begin{tabular}{|l|l|}
\hline & $(\%)$ \\
\hline Burnout élevé & 6,6 \\
\hline Burnout modéré & 19,7 \\
\hline Burnout léger & 25 \\
\hline
\end{tabular}

Nous avons souhaité étudier les corrélations existantes entre ce niveau de risque de burnout chez les enseignants et le niveau de décrochage professionnel. Le test statistique calculé entre les trois niveaux de risques léger, modéré et élevé du test MBI du Maslash et l'indice de décrochage calculé à partir de l'échelle construite montrent un lien statistique entre le risque de décrochage (moyen, silencieux ou visible) et le burnout élevé et modéré avec une valeur-p de $<0,0001$.

Tableau 11 : Test de corrélation (Pearson) entre les variables de burnout et les niveaux de décrochage

\begin{tabular}{|l|l|l|l|}
\hline Variables & $\begin{array}{l}\text { Décrochage au-dessus } \\
\text { de la moyenne }\end{array}$ & $\begin{array}{l}\text { Décrochage silencieux au- } \\
\text { dessus de la moyenne }\end{array}$ & $\begin{array}{l}\text { Décrochage visible au- } \\
\text { dessus de la moyenne }\end{array}$ \\
\hline $\begin{array}{l}\text { Burnout } \\
\text { élevé }\end{array}$ & $\mathbf{0 , 1 9 5}$ & $\mathbf{0 , 1 9 8}$ & $\mathbf{0 , 2 3 6}$ \\
\hline $\begin{array}{l}\text { Burnout } \\
\text { modéré }\end{array}$ & $\mathbf{0 , 1 9 7}$ & $\mathbf{0 , 2 1 1}$ & $\mathbf{0 , 0 9 7}$ \\
\hline $\begin{array}{l}\text { Burnout } \\
\text { léger }\end{array}$ & 0,066 & $\mathbf{0 , 1 4 3}$ & 0,081 \\
\hline Les valeurs en gras sont différentes de 0 à un niveau de signification alpha $=0,05$ & \\
\hline
\end{tabular}

Le calcul des odds ratios entre ces variables montre une relation forte entre les variables. 


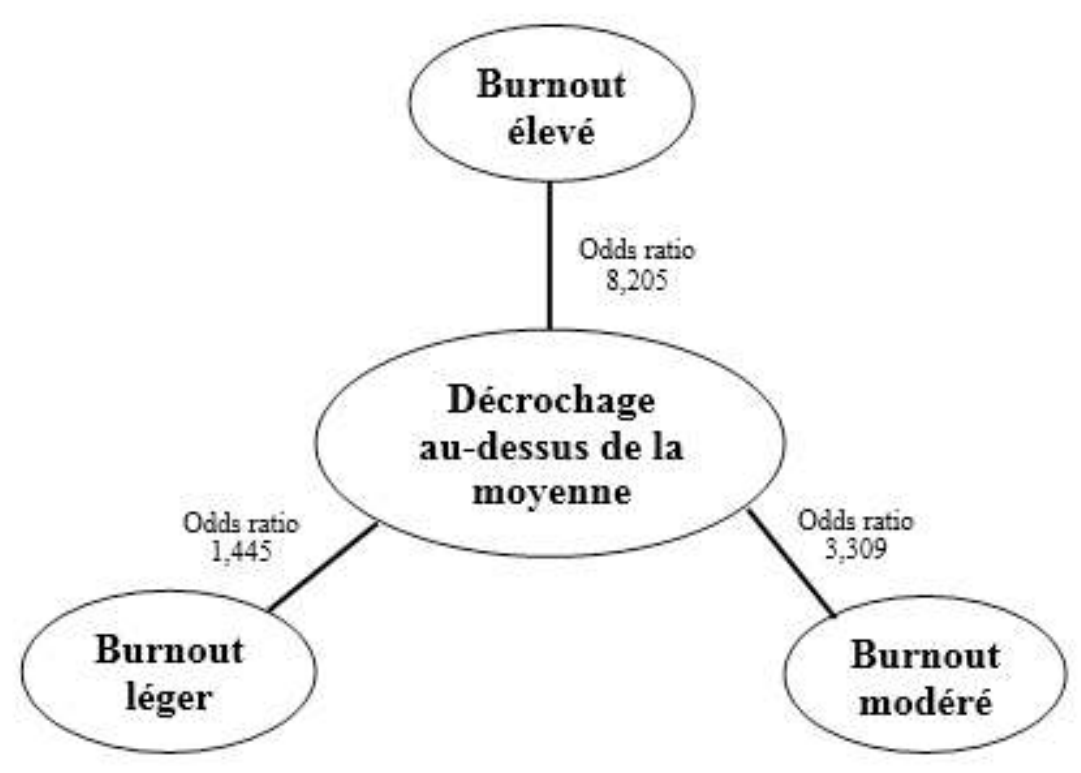

\section{3. Échelle de Karasek}

Le questionnaire de Karasek, principal outil d'évaluation des facteurs de risques psychosociaux au travail, permet d'évaluer globalement la santé mentale des professionnels. La version du questionnaire utilisé dans l'enquête Sumer internationale en 2003 comporte 26 questions.

41 Le questionnaire de Karasek mesure 4 dimensions (la latitude décisionnelle, la demande psychologique, le soutien social, la reconnaissance).

1. La latitude décisionnelle qui est la possibilité de choisir sa façon de travailler, de participer aux décisions qui s'y rattachent.

2. La demande psychologique qui est la charge psychologique associée à l'exécution des tâches, à la quantité et à la complexité des tâches, aux tâches imprévues, aux contraintes de temps, aux interruptions et aux demandes contradictoires.

3. Le soutien social qui est l'aide et la reconnaissance des collègues et de la hiérarchie.

4. La reconnaissance qui est le sentiment d'être reconnu dans son travail et dans les efforts professionnels qu'on déploie.

Une situation de travail est génératrice de stress si elle associe des exigences élevées au niveau du travail, peu ou pas de contrôle sur son propre travail et un soutien social faible de la part de l'équipe de travail ou de la hiérarchie.

Dans notre enquête, nous avons, à partir de la même grille de recueil, mesuré dans notre population l'ensemble des dimensions du test.

Tableau 12 : Tri à plat des dimensions du teste Karasek

\begin{tabular}{|l|l|l|}
\hline Échantillon & Modalité & $(\%)$ \\
\hline \multirow{2}{*}{ autonomie décisionnelle } & Non & 46,0 \\
\cline { 2 - 3 }
\end{tabular}




\begin{tabular}{|c|c|c|}
\hline & \\
\hline & Oui & 54,0 \\
\hline \multirow{2}{*}{ utilisation des compétences } & Non & 44,4 \\
\hline & Oui & 55,6 \\
\hline \multirow{2}{*}{ latitude décisionnelle } & Non & 49,2 \\
\hline & Oui & 50,8 \\
\hline \multirow{2}{*}{ soutien social de la hiérarchie } & Non & 50,0 \\
\hline & Oui & 50,0 \\
\hline \multirow{2}{*}{ soutien social des collègues } & Non & 51,6 \\
\hline & Oui & 48,4 \\
\hline \multirow{2}{*}{ soutien social global } & Non & 51,6 \\
\hline & Oui & 48,4 \\
\hline \multirow{2}{*}{ reconnaissance au travail } & Non & 57,3 \\
\hline & Oui & 42,7 \\
\hline \multirow{2}{*}{ demande psychologique } & Non & 50,8 \\
\hline & Oui & 49,2 \\
\hline
\end{tabular}

La combinaison des dimensions de la latitude décisionnelle et de la demande psychologique permet de mesurer le niveau de stress des individus.

Schéma 3 : Répartition des sujets suivant le niveau de stress (Karasek)

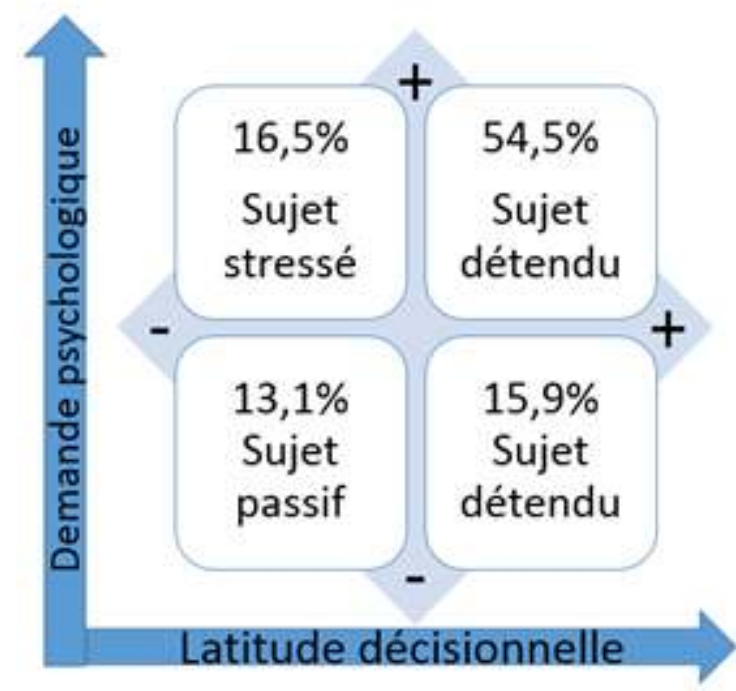

Nous avons identifié une population à risque de retrait professionnel à partir des données suivantes :

- Job-strain (niveau de stress élevé $16,5 \%$ de la population),

- Iso-strain (stress élevé et niveau faible du soutien social 10,1 \% de la population.

L'enquête Dares Sumer 2010 indique que 11,8 \% des professionnels cadres et professions intellectuelles sont des sujets stressés ce qui est nettement en dessous de notre enquête. 
Lamti, (2013) dans une étude portant sur les enseignants de collèges indique que $18 \%$ de ces enseignants ont un niveau de stress élevé. Notre enquête montre un taux de Job strain pour les enseignants de collèges de $22,2 \%$

Tableau 13 : Croisement entre le type d'établissement et les niveaux de stress

\begin{tabular}{|l|l|l|l|}
\hline & Primaire & College & Lycée \\
\hline Détendu & 15,2 & 18,5 & 0,0 \\
\hline Passif & 8,7 & 11,1 & 33,3 \\
\hline Actif & 58,7 & 48,1 & 66,7 \\
\hline Stresse & 17,4 & 22,2 & 0,0 \\
\hline
\end{tabular}

47 Pour poursuivre notre étude, nous avons cherché à repérer des liens entre le niveau de stress des enseignants et l'échelle de décrochage professionnel. Le test de corrélation et le test de Fischer nous permettent d'étudier les corrélations existantes entres ces variables.

Tableau 14 : Test de Pearson entre les niveaux de décrochage et les variables du test Karasek

\begin{tabular}{|l|l|l|l|}
\hline Variables & $\begin{array}{l}\text { Décrochage au } \\
\text { dessus de la } \\
\text { moyenne }\end{array}$ & $\begin{array}{l}\text { Décrochage silencieux au } \\
\text { dessus de la moyenne }\end{array}$ & $\begin{array}{l}\text { Décrochage visible au } \\
\text { dessus de la moyenne }\end{array}$ \\
\hline $\begin{array}{l}\text { Reconnaissance au } \\
\text { travail }\end{array}$ & $\mathbf{0 , 2 6 1}$ & $\mathbf{0 , 4 1 2}$ & $\mathbf{0 , 1 4 6}$ \\
\hline Soutien social social & $\mathbf{0 , 3 1 2}$ & $\mathbf{0 , 2 5 9}$ & $\mathbf{0 , 2 5 3}$ \\
\hline $\begin{array}{l}\text { Soutien } \\
\text { collègues }\end{array}$ & $\mathbf{0 , 3 1 2}$ & $\mathbf{0 , 2 5 9}$ & $\mathbf{0 , 2 5 3}$ \\
\hline $\begin{array}{l}\text { Soutien } \\
\text { hiérarchie }\end{array}$ & $\mathbf{0 , 3 2 2}$ & $\mathbf{0 , 3 2 3}$ & $\mathbf{0 , 2 6 9}$ \\
\hline $\begin{array}{l}\text { Utilisation } \\
\text { compétences }\end{array}$ & $\mathbf{0 , 1 9 6}$ & $\mathbf{0 , 4 0 2}$ & $\mathbf{0 , 2 7 1}$ \\
\hline $\begin{array}{l}\text { Autonomie } \\
\text { décisionnelle }\end{array}$ & $\mathbf{0 , 2 2 2}$ & $\mathbf{0 , 3 7 0}$ & $\mathbf{0 , 1 9 4}$ \\
\hline Niveau de stress & & $\mathbf{0 , 1 1 1}$ \\
\hline Les valeurs en gras sont différentes de 0 à un niveau de signification alpha =0,05 & \\
\hline
\end{tabular}

Le test de Fischer permet de calculer l'Odds ratio entre les variables. Il montre un niveau élevé des relations entre le type de stress des enseignants et les niveaux de décrochage professionnel. 
Tableau 15 : Croisement entre les niveaux de décrochage et les catégories du test Karasek

\begin{tabular}{|l|l|l|l|l|l|}
\hline & Détendu & Passif & Actif & Stressé & Odds ratio \\
\hline Décrochage & 6,8 & 13,6 & 61,4 & $\mathbf{1 8 , 2}$ & 22,2 \\
\hline Décrochage silencieux & 4 & 16 & 60 & $\mathbf{2 0}$ & 55 \\
\hline Décrochage visible & 9,8 & 13,7 & 56,9 & $\mathbf{1 9 , 6}$ & 13,3 \\
\hline Population générale & 14,6 & 12,2 & 56,1 & 17,1 & \\
\hline
\end{tabular}

49 Nous constatons que, chez les enseignants placés dans la catégorie décrochage, il y a plus d'enseignants stressés (test Karasek) que dans la population globale. Nous pouvons aussi constater que pour les détendus il existe moins de décrocheurs que dans la population globale. De plus nous remarquons que le risque (odds ratio) d'être décrocheur chez les enseignants stressés est 22,2 fois plus important que si on est dans une autre catégorie. Le risque est 55 fois plus important si l'on observe le décrochage silencieux.

\section{Conclusion}

50 Face aux difficultés scolaires au sein des établissements, les enseignants mettent en œuvre des stratégies professionnelles d'adaptation et de réponses. Compte tenu de la demande sociale, de l'évolution des publics, des transformations des métiers, les enseignants éprouvent des difficultés à adapter leurs manières de faire et leurs actes. Ils éprouvent alors stress, fatigue et sentiment d'empêchement à agir. Face à la détérioration de leur qualité de vie au travail, un certain nombre d'enseignants perçoit une perte de leur capacité à agir et éprouve la nécessité de prendre du recul ou de changer leurs manières de faire. Le décrochage professionnel des enseignants qu'il soit silencieux ou visible est un mode de réponse active à des changements ou des fragilisations des conditions de vie et de relation professionnelle. Les résultats de notre étude prolongent certaines recherches qualitatives sur le décrochage professionnel (Hernandez \& Méard, 2014 ; Zimmermann \& Méard 2015) et en même temps permettent d'identifier les items de ce décrochage professionnel et de mesurer son niveau, notamment l'incapacitation perçue par l'enseignant qui est un élément principal dans l'activité de retrait professionnel corrélé avec le décrochage professionnel.

51 Enfin, notre recherche montre que les dimensions du décrochage sont corrélées avec le niveau de stress professionnel mesuré d'après l'échelle Karasek et avec le risque de burnout de l'échelle Maslach MBI. Ces résultats confirment les travaux de sociologie du travail montrant les liens entre le stress professionnel et les procédures de « retrait professionnel défensif» dans de nombreux métiers en tension (policiers, gardiens de prison, infirmiers) (Monjardet, 2005 ; Bougard, 1997 ; Théry, 2006 ; Molinier, 2010). Des études complémentaires devront être conduites pour valider l'échelle du décrochage utilisée et mesurer les éléments qui favorisent son apparition. D'autres analyses plus sociologiques devraient nous aider à caractériser les variables objectives de carrière, de climat scolaire, de conditions de travail pour mieux prévenir ces situations de décrochage professionnel chez les enseignants. 


\section{BIBLIOGRAPHIE}

Altet, M. (2002). Une démarche de recherche sur la pratique enseignante : l'analyse plurielle. Revue française de pédagogie, 138(1) 85-93.

Barrère, A. (2002). Les enseignants au travail. Routines incertaines. Revue française de pédagogie, 140(1). 133-134.

Basco, L. (2003). Le malaise des enseignants du premier degré. Recherches et Education, $\mathrm{N}^{\circ} 4,1 \mathrm{er}$ trimestre 2003.

Blin, J.F. (2001). Classes difficiles, des outils pour prévenir et gérer les perturbations scolaires. Paris: Delagrave.

Bolin, F. S. (1989). Empowering leadership. Teacher College Record 91(1 Fall), 81-96.

Borg, M. (1990). Occupational stress in British educational settings: A review. Educational Psychology, 10, 103-126.

Borman, G. D., \& Dowling, N. M. (2008). Teacher attrition and retention: A meta-analytic and narrative review of the research. Review of Educational Research, 78(3), 367-409.

Bougard, D. (1997). Prévenir et remédier à l'usure professionnelle des personnels sanitaires et sociaux. Gestions hospitalières, 365, 274-278.

Bressoux, P. (1994). Les recherches sur les effets-écoles et les effets-maîtres. Revue Française de Pédagogie, 108, 91-137.

Bru, M. (1992). Les variations didactiques dans l'organisation des conditions d'apprentissage. Toulouse : Éditions Universitaires du Sud.

Bru, M., \& Maurice, J.J. (2001). Les pratiques enseignantes : contributions plurielles. Les dossiers des sciences de l'éducation, $\mathrm{N}^{\circ} 5$, Toulouse : PUM.

Chaplain, R. P. (2008). Stress and psychological distress among trainee secondary teachers in England. Educational Psychology, 28(2), 195-209.

Davies, I., Fulop, M., Hutchings, M., Ross, A., \& Berkics, M. (2004). Citizenship and enterprise: Issues from an investigation of teachers' perceptions in England and Hungary. Comparative Education, 40 (3), 363-384.

Debarbieux E. (1996). La violence dans la classe. ESF Editeur.

Debarbieux E, Blaya C. (2009). Le contexte et la raison : agir contre la violence à l'école par « l'évidence » ?. Revue criminologie. Volume 42, Numéro 1. pp. 13-31

Debarbieux E, Fotinos G. (2011). Violence et climat scolaire dans les établissements du second degré en France. Une enquête quantitative de victimation auprès des personnels de direction des lycées et collèges. Bordeaux : Observatoire international de la violence à l'école : CASDEN.

Dunham, J. (1992). Stress in teaching. London : Routledge.

Duru-Bellat M. \& Mingat A. (1997), La constitution des classes de niveau dans les collèges. Les effets pervers d'une pratique à visée égalisatrice. Revue Française de Sociologie, XXXVIII-4, 759-789. 
Farber, B. A. (1984). Stress and burnout in suburban teachers. Journal of Educational Research, 77(6), 325-331.

Galloway, D., Panckhurst, F., Boswell, K., \& Green, K. (1984). Mental health, absence from work, stress and satisfaction in a sample of New-Zealand primary school teachers. Australian and New Zealand Journal of Psychiatry, 18, p. 359-363.

Guarino, C., Santibanez, L., \& Daley, G. (2006). Teacher recruitment and retention: A review of the recent empirical literature. Review of Educational Research, 76(2), 173-208.

Gendron, B. (2008). Capital émotionnel en éducation. Dictionnaire de l'éducation dirigé par Van Zanten. Paris : PUF.

Hernandez, P., Méard, J. (2014). Développer le collectif de travail pour lutter contre le décrochage professionnel : le cas des cadres d'éducation dans l'enseignement privé. Questions vives Recherches en éducation, 21.

Huberman, M. (1993). Burnout in teaching Careers. European Education, 25 (3), 47- 69.

Ingersoll, R. (2001). Teacher turnover and teacher shortages: An organizational analysis. American Educational Research Journal, 38(3), 499-534.

Janot, L. (2005). Réactions émotionnelles et cognition des situations stressantes chez les enseignants d'école primaire. Éduquer, $\mathrm{N}^{\circ} 10.32-45$.

Jaoul, G., Kovess, V. (2004). Le burnout dans la profession enseignante. Annales Médico Psychologiques, 162, 26-35.

Karasek R., Brisson C., Kawakami N., Houtman I., Bongers P., \& Amick B. (1998). The Job Content Questionnaire (JCQ): An Instrument for Internationally Comparative Assessments of Psychosocial Job characteristics. Journal of Occupational Health Psychology. vol. 3, $\mathrm{N}^{\circ} 4,322-355$.

Karsenti, T., \& Collin S., (2009), L'autre décrochage scolaire, Formation et profession, Mars 2009, 2.

Karsenti, T., Collin, S., Villeneuve, S., Dumouchel, G., \& Roy, N. (2008). Pourquoi les nouveaux enseignants d'immersion ou de français langue seconde quittent-ils la profession? Résultats d'une enquête pancanadienne. Ottawa, ON : Association canadienne des professeurs d'immersion.

Kirsch, R. (2006). L'abandon volontaire de la carrière chez des enseignants débutants du primaire et du secondaire au Québec. Mémoire de maitrise non publie, Université de Montréal, Montréal, QC, Canada.

Kyriacou, C. (1987) Teacher stress and burnout: An international review. Educational Research, 29 (2), 145-152.

Laplante J., \& Tougas F. (2011). La privation relative et le niveau d'identification comme déclencheurs du désengagement psychologique : une étude exploratoire auprès d'éducatrices, Les Cahiers Internationaux de Psychologie Sociale, $\mathrm{N}^{\circ}$ 89-90, 43-61.

Laugaa, D. (2004). Stress et burnout des enseignants en école élémentaire. Une approche transactionnelle. Thèse de doctorat en Psychologie, Université de Bordeaux 2.

Laughlin, A. (1984). Teacher stress in an Australian setting: the role of biographical mediators. Educational Studies, 10, 7-22.

Lecuivre, G. \& Roger, J.L. (2012). Empêchements de l'actvité professionnelle et pouvoir d'agir. Repéré à http://www.ices.fr/BU/documents/ koha_99956/atel/atel_2.htm.

Macdonald, D. (1999). Teacher attrition: A review of literature. Teaching and Teacher Education, 15 (8), 835-848. 
Maleyrot E. (2002). Ruptures et transformations identitaires des maîtres formateurs face aux réformes de la formation des enseignants, Les Sciences de l'Éducation pour l'Ère Nouvelle, 45(3), 65-88.

Maslach, C., Jackson, S. E., \& Leiter, M. P. (1996). The Maslach Burnout Inventory (3rd ed.). Palo Alto, CA : Consulting Psychologists Press.

Molinier, P. (2010). Souffrance, défenses et reconnaissance. Le point de vue du travail. Nouvelle revue de psychosociologie, 10, 99-110.

Monjardet D. (2005). « Gibier de recherche. La Police et le projet de connaître ». Criminologie, vol. XXXVIII, $\mathrm{n}^{\circ} 2$.

Montoya Y., \& Debarbieux E. (1998). La violence à l'école en France : 30 ans de construction sociale de l'objet (1967-1997), Revue française de Pédagogie, 123.

Morcilla, A., Martin, F., \& Blin, J.F. (2004). Le vécu émotionnel des enseignants confrontés à des perturbations scolaires. Revue des sciences de l'éducation, Volume 30, $\mathrm{N}^{\circ} 3,579-604$.

Ndoreraho, J.-P. \& Martineau, S. (2006). Une problématique des débuts de la carrière en enseignement. Trois-Rivières, UQTR, Repéré à http://www.insertion.qc.ca/IMG/pdf/NdorerahoMartineau-2006-Une-proble_matique.pdf

Ourghanlian C. (2011). Je veux qu'on me change. La lettre de l'enfance et de l'adolescence, 1(83-84), 95-102.

Pécout, E., \& Méard, J. (2014). L'usage des TIC en classe de $4^{\mathrm{e}}$ pour accrocher les élèves. In E. Flavier \& S. Moussay. Répondre au décrochage scolaire : expériences de terrain. Bruxelles : De Boeck. Romano, M. (2008). Successes and struggle of the beginning teacher: Widening the sample. The Educational Forum, 72(1), 63-78.

Rudow, B. (1999). Stress and burnout in the teaching profession: european studies, issues and research perspectives. In Vandenberghe, R., \& Huberman, A. M. (Eds.) Understanding and preventing teacher burnout (pp. 38-58). Cambridge University Press.

Smylie, M. A. (1999). Teacher stress in a time of reform in teaching. In Vandenberghe, R., \& Huberman, A. M. (Eds.), Understanding and preventing teacher burnout (pp. 59-84). Cambridge University Press.

Sprague, J. (1992). Critical perspectives on teacher empowerment. Communication Education, 41(2), 181-203.

Stoel, C. F., \& Thant, T.-S. (2002). Teachers' professional lives - A view from nine industrialized countries . Washington, DC: Milken Family Foundation.

Talbert, T. L. (2003). Come to the edge: Embracing teacher empowerment for the 21st century. Action in Teacher Education, 25(2), 51-55.

Théry, L. (2006). Introduction. In L. Théry (Ed). Le travail intenable. Résister collectivement à l'intensification du travail. Paris, La Découverte.

Van Horn, J. E., Schaufeli, W. B., Greenglass, E. R., \& Burke, R. J. (1997). A Canadian - Dutch comparison of teachers' burnout. Psychological Reports, 81, 371-382.

Vergne, G. (2010). De l'aliénation enseignante. Revue Skole. Repéré à http://skhole.fr/de-l-ali\% C3\%A9nation-enseignante.

Wisniewski, L., \& Gargiulo, R. (1997). Occupational stress and burnout among special educators: A review of the literature. The Journal of Special Education, 31, 325-346. 
Woods, P. (1977). Teaching for survival. In P. Woods \& M. Hammersley (eds), School Experience Explorations in the Sociology of Éducation, London: Croom Helm, 271-293.

Yamashita, 2006. The relationship between the teachers' teaching styles, students' engagement in school and ESL. Mise en ligne http://titaproject.eu/spip.php?article132.

Zimmermann, P. \& Méard, J. (2015) L'accrochage professionnel des enseignants novices face à des élèves en situation de décrochage scolaire. Education \& Formation, e-304

\section{RÉSUMÉS}

Au sein de ses pratiques professionnelles, l'enseignant gère des situations stressantes, difficiles et fatigantes; la capacité de l'enseignant à gérer ces situations est au cœur de recherches des années 1990 portant sur l'action enseignante. Dans certaines conditions, ces situations perturbent le professionnel et ont une incidence notable sur sa santé et son comportement. L'enseignant alors peut décrocher de la situation ou développer des sentiments et des actes montrant son incapacité à agir (disempowerment). Dans le cadre d'une étude sur les modalités de gestion par l'enseignant des situations de décrochage scolaire conduites auprès de 640 enseignants de Midi-Pyrénées, nous avons identifié et validé une échelle du décrochage enseignant et nous avons montré les liens entre cette échelle de pratiques et l'état de fatigue, de stress et de mal-être de l'enseignant.

Within a teacher's professional practice, it is often that he/she will handle stressful situations, being both difficult and tiring. Teacher's ability to handle these situations is at the heart of research on teacher action since the early nineties. In several circumstances, these situations can disturb the professionals and have a significant impact on their health and behavior. In such cases, the teacher can either just drop out of the situation or develop feelings and behaviors showing its inability to act (disempowerment). As part of a study on the modalities of teacher management of school dropout situations conducted among 640 teachers from Midi-Pyrénées (France), we identified and validated a scale for assessing teacher attrition and we showed the links between this scale of attrition practices and the general level of fatigue, stress and discomfort experienced by the teacher.

\section{INDEX}

Keywords : teacher attrition, teacher action, burnout, empowerment, discomfort

Mots-clés : décrochage enseignant, action enseignante, burnout, empowerment, mal-être 